\title{
Gene Silencing - Targeting at Gene Methylation and Demethylation Patterns for the Arrest of Cancer Progression
}

\section{Rekha Govindan ${ }^{1 *}$, Sourabh Suran ${ }^{2}$ and Sanjeeva Metikala ${ }^{3}$}

${ }^{1}$ Department of Biotechnology, Aarupadai Veedu Institute of Technology, Vinayaka Missions University, India ${ }^{2}$ CSIR-Centre for Cellular and Molecular Biology, Uppal Road, Hyderabad 500007, Andhra Pradesh, India ${ }^{3}$ Institute for Physiological Chemistry, Martin Luther University, Halle-Wittenberg, Germany

\begin{abstract}
The sudden occurrence or silent recurrence of tumor has been found to result from long term molecular changes at DNA level, that eventually lead to the abnormal expansion of cancer cells. One such molecular change is accounted by a distinct promoter DNA hyper-methylation pattern in certain tumor suppressing genes that are usually the check points for the regulation of gene expression in normal and progenitor cells. The hyper methylated promoter regions may account for the transcriptional inactiveness of the tumor suppressor genes. This possibility, coupled with the reversible nature of epigenetics such as demethylation has enormous significance for the prevention and control of cancer. In such circumstances, the use of demethylating drugs may re-express those genes that were silenced by aberrant DNA methylation. Understanding these epigenetic changes may help in developing specific markers to identify the molecular pathways of tumorigenesis and for deriving sensitive molecular detection strategies for a variety of tumor type.
\end{abstract}

Keywords: Gene silencing; DNA methylation; De-methylation; Diagnostic markers for cancer

\section{Introduction}

DNA methylation involves covalent addition of methyl groups to the cytosine pyrimidine ring or to the adenine purine ring. More commonly the addition occurs in cytosine within $\mathrm{CpG}$ dinucleotides which are concentrated in large clusters called $\mathrm{CpG}$ islands within the regulatory regions of genes, where transcription is initiated. In bulk of genomic DNA the CpG sites within the promoter regions are heavily methylated allowing a strict regulation of gene transcription process. The methyl groups project into the major grooves of DNA and causes steric hindrance for the RNA polymerase thereby inhibiting the initiation of gene transcription. This is identified as one of the major epigenetic changes involved in the transcriptional inactivation of certain tumor-suppressor genes leading to sudden occurrence or silent recurrence of tumors [1-3].

DNA methylation is an enzymatic process occurring during the normal organismal development and cellular differentiation in higher organisms. It is also one of the major epigenetic mechanisms involved in regulating the chromatin structure and gene expression. The methylation pattern is different in cells where certain genes are to be constitutively expressed. Normally such cells belong to the germ line cells and their $\mathrm{CpG}$ islands remain unmethylated, allowing constitutive gene expression to occur [3-5]. In normal somatic cells, DNA methylation helps to maintain transcriptional silence in the noncoding regions of the genome. Considering the pericentromeric heterochromatin that is transcriptionally inactive and hyper-methylated, the role of DNA methylation may be to ensure that such junk DNA is to replicate at a later stage diverting the energy for the transcription of the house keeping genes. There is also a chance that such mechanism involves a defensive role in suppressing the expression of any potentially harmful viral sequences or transposons that may have integrated into such sites $[2,6,7]$.

\section{Abnormal DNA methylation in cancer occurrence and recurrence}

Tumorigenesis arises as a consequence of two related events, one is the increased activity of agents or factors that promote uncontrolled cell differentiation and the other is the decreased activity of factors that suppress the uncontrolled cell differentiation. Additional abnormalities like regulation of cell-cell and cell matrix attachment, growth factor signalling, apoptosis and recombination must accompany the change in differentiative capacity to cause tumorigenesis $[8,9]$.

DNA methylation is one of the widely studied epigenetic modifications due to its significant role in gene expression and silencing. Changes from the normal DNA methylation patterns are observed in cancer occurrence and recurrence. One is an overall genome-wide reduction in DNA methylation (global hypomethylation) and the other is regional hypermethylation within the $\mathrm{CpG}$ islands of specific gene promoters. Global hypomethylation results in the induction of protooncogene activation and chromosomal instability, whereas regional hypermethylation especially within the regulatory sequences is strongly associated with transcriptional silencing of tumor suppressor genes $[8,10]$.

Even though there are many number of pathways altered during the process of tumorigenesis, the processes linked to DNA methylation provide potential mechanisms leading to heritable genomic changes that promote cancer. In such cells, methylation within promoters serves to turn off critical genes that could otherwise suppress tumorigenesis. This is demonstrated by the hypermethylation of tumor suppressor genes, those encoding cell adhesion molecules and growth-regulatory

*Corresponding author: Rekha Govindan, Associate Professor, Department of Biotechnology, Aarupadai Veedu Institute of Technology, Vinayaka Missions University, Rajiv Gandhi Salai, Paiyanoor, Kanchipuram, Tamil Nadu - 503604 India, Tel: 09566150994; E-mail: rekhagovindan2000@yahoo.com

Received March 17, 2014; Accepted April 25, 2014; Published April 28, 2014

Citation: Govindan R, Suran S, Metikala S (2014) Gene Silencing - Targeting at Gene Methylation and Demethylation Patterns for the Arrest of Cancer Progression. J Cancer Sci Ther 6: 174-176. doi:10.4172/1948-5956.1000268

Copyright: @ 2014 Govindan R. This is an open-access article distributed under the terms of the Creative Commons Attribution License, which permits unrestricted use, distribution, and reproduction in any medium, provided the original author and source are credited. 
proteins that are often silenced in hematopoietic malignancies. Many of these genes play a role in regulating cell growth, differentiation, signal transduction, and DNA repair, while others are implicated in tumor metastasis and angiogenesis. Almost half of the tumor suppressor genes carrying germ line mutations in familial cancer are due to the inactivation in association with CpG island hypermethylation $[4,8,9]$.

\section{Reversal of epigenetic changes and arrest of cancer progression by pharmacologic agents}

It has been said that at the molecular level, genetic and epigenetic modifications contribute to cancer development and progression. While the genetic changes are not reversible, epigenetic changes can return to their normal state. Such reversible changes have been the focus of treatments offered by epigenetic drugs for the inhibition of formation and growth of cancer progenitor cells, thus reducing the recurrence of cancer. As methylation of $\mathrm{CpG}$ islands occurs infrequently in normal cells, it provides a selective tumor-specific therapeutic target. Pharmacologic inhibition of methylation-mediated suppression could therefore derepress inappropriately silenced genes and restore normal gene function [11-15].

Re-expression of hypermethylated genes in cancer cells is conducted by demethylating drugs. Many epigenetic drugs have been discovered in the recent past that can effectively reverse DNA methylation that occurs in cancer. DNA methylation inhibitors such as 5-azacytidine (5-aza-CR) and 5-aza2'-deoxycytidine (5-aza-CdR) were among the first epigenetic drugs proposed for use as cancer therapeutics. The DNA demethylating drugs azacitidine and its deoxy derivative, decitabine, are powerful inhibitors of DNA methylation. After cellular uptake and phosphorylation, azacitidine is incorporated into RNA, where it suppresses RNA synthesis and has cytotoxic effects. After conversion to 5-aza-2'-deoxycytidine diphosphate by ribonucleotide reductase and subsequent phosphorylation, the triphosphate form is incorporated into DNA in place of the natural base cytosine. Because of the substitution of the 5' nitrogen atom in place of the carbon, the DNMTs are trapped on the substituted DNA strand and methylation is inhibited. Thus, a significant proportion of the DNA becomes hemimethylated. A second round of DNA synthesis in the presence of these drugs results in full double-stranded DNA demethylation $[2,14]$.

Inhibitors of DNA methylation can reverse epigenetic changes in a number of target genes that have a role in the development and/or progression of leukemia's. Clinical studies clearly demonstrated that such agents used alone or in combination, have clinical benefit in many patients with hematologic malignancies. These results strongly suggest that the clinical activity of agents such as azacitidine and decitabine is mediated by targeting reversal of gene silencing. However a formal proof of this causal relationship and identification of the critical gene targets are still lacking. Future research will undoubtedly provide this evidence and suggest additional therapeutic targets regulating methylation and histone acetylation pathways in cancer $[2,15]$.

\section{DNA methylation status as diagnostic and prognostic index for tumor development}

DNA methylation is one of the major aberrant epigenetic mechanisms that influence the transcription of genes important to the cancer process. This in turn suggests that DNA methylation may hold a key to understanding the origins of cancer epigenetic changes. With the overwhelming importance of aberrant DNA methylation especially in $\mathrm{CpG}$ islands of genes involved in tumorigenesis coupled with the reversible nature of epigenetics, such molecular changes can be used as indicative markers for early cancer detection, disease progression, and prediction of the response to cancer treatment $[16,17]$.

14-3-3sigma is an epithelial marker whose expression is induced by DNA damage through a p53-dependent pathway. 14-3-3sigma function sequesters cyclin $\mathrm{B} 1-\mathrm{CDC} 2$ complexes outside the nucleus and thereby contributes to a G2 arrest. Hypermethylation of the 143-3 sigma promoter is an early and frequent event in breast neoplastic transformation, leading to the suggestion that silencing of 14-3-3 sigma may be an important event in tumor progression and particularly in breast carcinogenesis $[18,19]$.

The over activation of HGF/cMET pathway has been implicated in several human malignancies. Genome-wide epigenetic screens on human medulloblastoma cell lines and primary tumor specimens have identified SPINT2/HAI-2, an HGF/cMET signalling inhibitor, as a novel tumor suppressor gene that is frequently silenced by promoter hypermethylation in medulloblastoma. The underlying clinical significance is that detection of CpG methylation of 14-3-3sigma or SPINT2/HAI-2 may be used for diagnostic and prognostic purposes $[20,21]$.

Methylation-sensitive restriction landmark genomic scanning involves digestion of genomic DNA with methylation-sensitive enzymes, followed by resolution of differentially digested fragments by two-dimensional gel electrophoresis. Comparison of gel migration profiles obtained from normal and tumor samples allows identification of aberrantly methylated loci. This technique was used to analyze the methylation status of $1184 \mathrm{CpG}$ islands in 98 primary human tumors. As suggested by studies of substantially smaller sets of $\mathrm{CpG}$ island loci, the results uncovered global non-random patterns of methylation in tumors, with specific methylation events associated with particular tumor types. There are many more techniques such as Microarray based approaches, PCR, Differential methylation hybridisation (DMH) to produce promising results towards the goals of cancer diagnostics, prognostics and therapeutics [22-24].

\section{Conclusion}

The biological significance of DNA methylation in the regulation of gene expression and its role in cancer is increasingly recognized. It is now clear that these epigenetic changes affect chromatin structure and thus regulate processes such as transcription, X-chromosome inactivation, allele-specific expression of imprinted genes, and inactivation of tumor suppressor genes. Further studies are needed to better characterize the interactions of the DNA methylation and histone deacetylation pathways, pinpoint the alterations in methylation that occur in normal development and cancer, and improve approaches to reverse inappropriate transcriptional silencing in tumor suppressor and other regulatory genes in cancer.

Treatment with inhibitors of DNA methylation and histone deacetylation can reactivate epigenetically silenced genes and has been shown to restore normal gene function. In cancer cells, this results in reexpression of tumor suppressor genes and other regulatory functions, inducing growth arrest and apoptosis. Azacitidine and decitabine are clinically active in leukemia and other hematologic malignancies and may have therapeutic potential in other malignant disorders. Continued clinical trials with hypomethylating agents and HDAC inhibitors, alone or in combination, may provide further advances in the treatment of hematopoietic malignancies and solid tumors. 
Citation: Govindan R, Suran S, Metikala S (2014) Gene Silencing - Targeting at Gene Methylation and Demethylation Patterns for the Arrest of Cancer Progression. J Cancer Sci Ther 6: 174-176. doi:10.4172/1948-5956.1000268

\section{References}

1. Lund $A H$, van Lohuizen M (2004) Epigenetics and cancer. Genes Dev 18 2315-2335.

2. Baylin SB (2005) DNA methylation and gene silencing in cancer. Nat Clin Pract Oncol 2 Suppl 1: S4-11.

3. Golbabapour S, Abdulla MA, Hajrezaei M (2011) A concise review on epigenetic regulation: insight into molecular mechanisms. Int J Mol Sci 12: 8661-8694.

4. Esteller M, Corn PG, Baylin SB, Herman JG (2001) A gene hypermethylation profile of human cancer. Cancer Res 61: 3225-3229.

5. Esteller M (2002) CpG island hypermethylation and tumor suppressor genes: a booming present, a brighter future. Oncogene 21: 5427-5440.

6. Morgan HD, Dean W, Coker HA, Reik W, Petersen-Mahrt SK (2004) Activationinduced cytidine deaminase deaminates 5-methylcytosine in DNA and is expressed in pluripotent tissues: implications for epigenetic reprogramming. $\mathrm{J}$ Biol Chem 279: 52353-52360.

7. Yagi S, Hirabayashi K, Sato S, Li W, Takahashi Y, et al. (2008) DNA methylation profile of tissue-dependent and differentially methylated regions (T-DMRs) in mouse promoter regions demonstrating tissue-specific gene expression. Genome Res 18: 1969-1978.

8. Suzuki H, Toyota M, Sato H, Sonoda T, Sakauchi F, et al. (2006) Roles and causes of abnormal DNA methylation in gastrointestinal cancers. Asian Pac Cancer Prev 7: 177-185.

9. Rivlin N, Brosh R, Oren M, Rotter V (2011) Mutations in the p53 Tumo Suppressor Gene: Important Milestones at the Various Steps of Tumorigenesis. Genes Cancer 2: 466-474.

10. Schoofs T, Berdel WE1, Müller-Tidow C2 (2014) Origins of aberrant DNA methylation in acute myeloid leukemia. Leukemia 28: 1-14

11. Li LC, Carroll PR, Dahiya R (2005) Epigenetic changes in prostate cancer: implication for diagnosis and treatment. J Natl Cancer Inst 97: 103-115.

12. Lande-Diner L, Zhang J, Ben-Porath I, Amariglio N, Keshet I, et al. (2007) Role of DNA methylation in stable gene repression. J Biol Chem 282: 12194-12200.
13. Mann M, Cortez V, Vadlamudi RK (2011) Epigenetics of estrogen receptor signaling: role in hormonal cancer progression and therapy. Cancers (Basel) 3: 1691-1707.

14. Ghavifekr Fakhr M, Farshdousti Hagh M, Shanehbandi D, Baradaran B (2013) DNA Methylation Pattern as Important Epigenetic Criterion in Cancer. Genet Res Int 2013: 317569.

15. Sarkar S, Horn G, Moulton K, Oza A, Byler S, et al. (2013) Cancer development progression, and therapy: an epigenetic overview. Int J Mol Sci 14: $21087-$ 21113.

16. Laird PW (2003) The power and the promise of DNA methylation markers. Nat Rev Cancer 3: 253-266.

17. Belinsky SA (2004) Gene-promoter hypermethylation as a biomarker in lung cancer. Nat Rev Cancer 4: 707-717.

18. Horie-Inoue K, Inoue S (2006) Epigenetic and proteolytic inactivation of 14-3-3sigma in breast and prostate cancers. Semin Cancer Biol 16: 235-239.

19. Martinez-Galan J, Torres-Torres B, Gonzalez-Vicente A, Ruiz-Vozmediano J, Delgado-Perez JR (2011) P1-05-06: Hypermethilation 14-3-3- Sigma Promoter as a Biomarker to Screening for Metastasis and Potencial Prognostic Factor in Breast Cancer Patients. Cancer Research 71: P1-05-06.

20. Peruzzi B, Bottaro DP (2006) Targeting the c-Met signaling pathway in cancer Clin Cancer Res 12: 3657-3660.

21. Kongkham PN, Northcott PA, Ra YS, Nakahara Y, Mainprize TG, et al. (2008) An epigenetic genome-wide screen identifies SPINT2 as a novel tumor suppressor gene in pediatric medulloblastoma. Cancer Res 68: 9945-9953.

22. Hatada I, Hayashizaki Y, Hirotsune S, Komatsubara H, Mukai T (1991) A genomic scanning method for higher organisms using restriction sites as landmarks. Proc Natl Acad Sci U S A 88: 9523-9527.

23. Ordway JM, Curran T (2002) Methylation matters: modeling a manageable genome. Cell Growth Differ 13: 149-162.

24. Pompeia C, Hodge DR, Plass C, Wu YZ, Marquez VE, et al. (2004) Microarray analysis of epigenetic silencing of gene expression in the KAS-6/1 multiple myeloma cell line. Cancer Res 64: 3465-3473. 